\title{
Pontine Infarct Camouflaged as Bell's Palsy
}

\author{
Kyle Phipps ${ }^{\mathrm{a}}$, Rajveer Joea ${ }^{\mathrm{b}, \mathrm{e}}$, Sarah J. Strube ${ }^{\mathrm{b}, \mathrm{c}}$, \\ Ignacio Carrillo-Nunez ${ }^{\mathrm{d}}$
}

\begin{abstract}
Facial palsy is a neurological disorder triggered by dysfunction of the seventh cranial nerve, categorized as either central, between the cerebral cortex and brainstem nuclei, or peripheral, between the brainstem nuclei and peripheral organs. Central lesions cause impairment of the contralateral lower facial musculature with associated sparing of the forehead and ocular muscles. Conversely, peripheral lesions produce ipsilateral whole-sided facial hemiplegia including both forehead and ocular muscles. Facial palsy is diagnosed clinically, while imaging studies and additional subsidiary testing (e.g. electromyography, nerve conduction studies), serologies, and rarely biopsy can assist in confirming or refuting the working diagnosis. The differential diagnosis comprises Bell's palsy (idiopathic), HIV infection, Ramsey Hunt syndrome, Lyme, sarcoidosis, amyloidosis, acoustic neuroma, parotid gland tumor, temporal bone biopsy, otitis media, Guillain-Barre syndrome, and brainstem infarct. Facial palsy is branded as Bell's palsy if one of the aforementioned etiologies is not identified as the root cause. Herein, we report the case of a 58-year-old male, who presented with left facial weakness involving both the upper and lower face, posterior circulation symptoms in the setting of hypertensive emergency. Initial magnetic resonance imaging (MRI) was unrevealing for any acute process, however given lack of improvement repeat imaging was ordered. The patient was ultimately confirmed to have an acute versus subacute pontine infarct where the seventh and eighth cranial nerve exits at the cerebellopontine angle. Ancillary laboratory studies were non-contributory. Subsequently, the patient's symptoms continued to improve, blood pressure control was achieved, and was consequently discharged on goal-directed medical therapy.
\end{abstract}

Keywords: Bell's palsy; Pontine; Brainstem; Infarct

Manuscript submitted February 8, 2019, accepted March 7, 2019

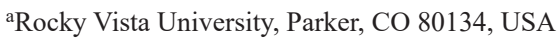

${ }^{b}$ Department of Internal Medicine, St. Mary Medical Center, Long Beach, CA 90813, USA

${ }^{c}$ Health Sciences, St. Mary Medical Center, Long Beach, CA 90813, USA ${ }^{\mathrm{d} D e p a r t m e n t ~ o f ~ N e u r o l o g y, ~ S t . ~ M a r y ~ M e d i c a l ~ C e n t e r, ~ L o n g ~ B e a c h, ~ C A ~} 90813$, USA

${ }^{e}$ Corresponding Author: Rajveer Joea, St. Mary Medical Center, GME, 1050 Linden Ave., Long Beach, CA 90813, USA. Email: rsjoea@gmail.com

doi: https://doi.org/10.14740/jnr524

\section{Introduction}

Bell's palsy, otherwise designated idiopathic peripheral facial nerve palsy being a diagnosis of exclusion, accounts for 60 $75 \%$ of all unilateral facial paralysis cases [1]. Classically, Bell's palsy is attributed to a herpes simplex-mediated viral inflammation with ensuing demyelination, and palsy. Unless genetically predisposed, risk factors for developing Bell's palsy are centered on eliciting facial nerve ischemia e.g. diabetes, pregnancy, hypercoagulability, and preeclampsia [2]. Accompanying symptoms may include taste disturbances involving two-thirds of the anterior tongue and xerostomia, dysacusis or hyperacusis, lagophthalmos, and decreased lacrimation resulting from the disrupted afferent and efferent functions of the facial nerve. Treatment entails early combination therapy with steroids and antivirals based on their House-Brackmann grade [3]; protective measures involving the cornea must also be addressed since patients may have concomitant lagophthalmos and reduced tearing.

Alternatively, an infarct arising in the ipsilateral facial nerve nucleus or facial nerve tract in the pons causes a lower motor neuron lesion mirroring Bell's palsy. A pontine stroke accounts for approximately one percent of all new facial paralysis cases [4]; Fahimi et al described a surveillance of nearly 44,000 Bell's palsy diagnoses in California emergency departments (ED) from 2005 to 2011, with less than one percent of patients obtaining an alternate diagnosis [5]. Imaginably, pontine infarcts are branded as idiopathic peripheral facial nerve palsy, albeit infrequently, with estimates suggesting one out of every 400 cases attributable to ischemic stroke $[5,6]$.

Pontine strokes characteristically arise as a result of occlusive lesions within the basilar artery $[7,8]$, whereas the anterior inferior cerebellar artery (AICA), a branch from the basilar artery, primarily supplies the facial nerve nucleus [9]. Small deep pontine infarcts are most likely the result of small vessel disease [8]. Infarcts within the facial nerve nucleus or its immediate outflow tract are responsible for central lesions that present with whole-sided facial weakness and are easily misdiagnosed as peripheral lesions. Stroke management differs considerably from that of Bell's palsy, with identification of stroke source and prevention of additional stroke taking immediate precedence in the patient's care after stabilization. Typically, this includes intracranial and extracranial vessels evaluation with computed tomography (CT) or MR angiography, cardiac evaluation via electrocardiogram (ECG) and transthoracic echocardiogram (TTE), swallow evaluation, blood pressure 


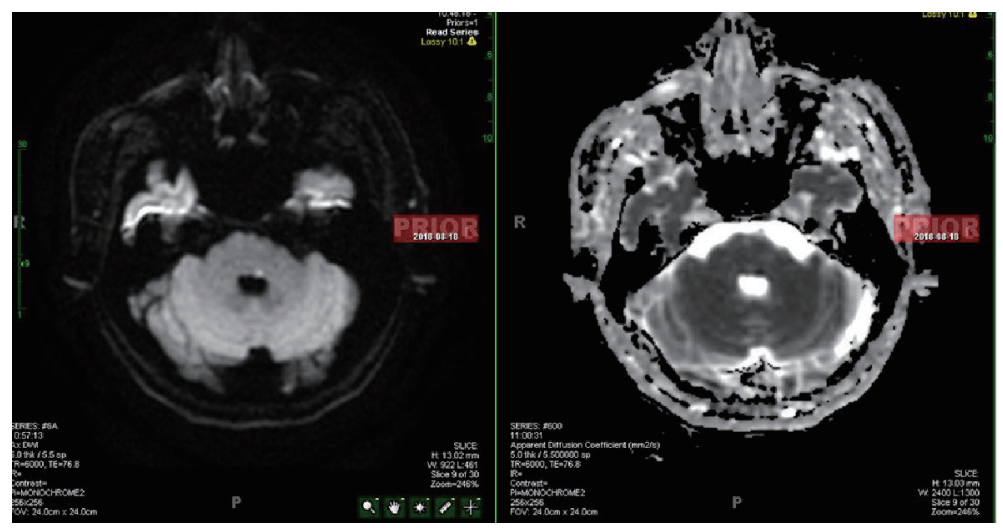

Figure 1. Punctate postero-parietal white matter acute infarct, old right centrum semi-ovale and basal ganglia infarcts shown by MRI on the day of admission.

control, antiplatelet, and statin therapy.

\section{Case Report}

A 58-year-old male presented to the ED with a 4-day history of dizziness, nausea, and vomiting. Moreover, he reported new onset diplopia and left-sided headache beginning earlier that morning, found to have a blood pressure of 206/110 mm $\mathrm{Hg}$ upon evaluation. Physical exam uncovered a left-sided facial droop with ptosis, but the patient remained unaware. By the same token, he was unable to raise his left eyebrow or to shut his left eyelid firmly, and had a diminished left nasolabial fold, and a droop of the left angle of the mouth when smiling. Other than left-sided facial weakness, no gross motor or sensory deficits were present. CT scan of the brain was negative for acute hemorrhage, while magnetic resonance imaging (MRI) revealed an acute small punctate infarct within the parietal lobe (Fig. 1). Evaluation of the posterior fossa was reported as unremarkable and the patient was subsequently admitted to the intensive care unit (ICU) for neurological monitoring and nicardipine drip.

The patient remained in the ICU for 2 days as his blood pressure stabilized before transferring to telemetry. During this time his diplopia, nausea, and vomiting resolved, though continued to report dizziness along with no change in his whole-left sided facial weakness. CT angiogram of the head and neck revealed a $30 \%$ stenosis of the left distal internal carotid artery; TTE with bubble study was unrevealing of a thrombotic source. Contrariwise, upon repeat evaluation of the original MRI of the brain, the consulting neurologist acknowledged a potential ischemic stroke at the exit of cranial nerve seven from the left pons that had not been previously reported. This finding in the setting of acute punctate infarcts found on MRI of the brain raised suspicion for vasculitis, although the workup was unconvincing - erythrocyte sedimentation rate 38 , rapid plasma reagin and antinuclear antibody negative. Hypertension-related stroke was presumptively diagnosed and the patient was continued on aspirin and atorvastatin.

After transferring to the medical floor, the patient continued to experience blood pressure fluctuations and worsening intermittent dizziness. A repeat MRI of the brain (Fig. 2) was ordered on the fifth day of admission to assess for the possibility of a new stroke or expansion of the recently diagnosed stroke. The formal read once again noted only small posteroparietal acute infarcts, nonetheless unchanged from prior. The case was further discussed with neurology, with specific attention given to the patient's atypical facial paralysis pattern and

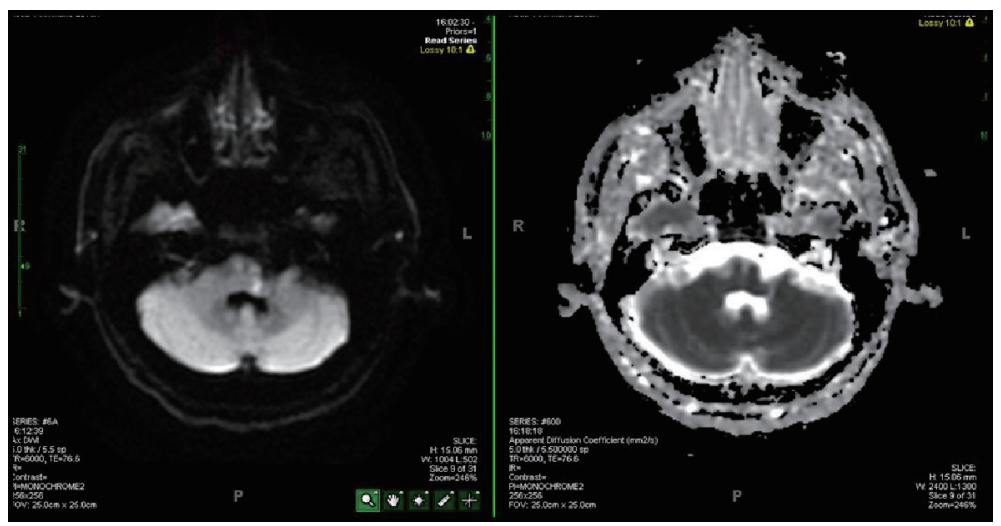

Figure 2. Acute vs. subacute left pontine infarct (DWI on the left, apparent diffusion coefficient (ADC) on the right) shown by MRI on the fifth day of admission. 
persistent dizziness. Upon further review of the subsequent MRI with the neurologist, a small left pontine stroke was confirmed near the exit of cranial nerves seven and eight. This finding correlated with the very small stroke evident on the original MRI that expanded and intensified in brightness on diffusion-weighted imaging (DWI).

\section{Discussion}

Ischemic stroke and Bell's palsy are the most common causes of facial hemiparesis or hemiplegia [1]. Differentiating between these two etiologies may seem straightforward in most cases, as the neurologic exam typically follows a central (upper motor neuron) or peripheral (lower motor neuron) lesion pattern. Cortical lesions present with weakness of only the lower two-thirds of the face, whereas peripheral lesion which is a lesion of the facial nerve itself present as total hemiplegia of the face, involving the eyelid and forehead. This phenomenon can be attributed to the various routes taken by nerves that provide motor innervation to the face. As motor cortex fibers innervating the lower face arrive in the brainstem, they decussate so that the right motor cortex exclusively and totally provides all motor function to the left lower face via the left facial nerve. Conversely, fibers that control the upper face take a different route. Upon reaching the brainstem, only half of these fibers decussate to the contralateral facial nerve nucleus, while half remain ipsilateral. This allows each hemisphere of the brain to provide bilateral innervation to the frontalis muscle, such that strokes in the motor cortex present with lower facial weakness only, while motor function of the forehead and eyes is preserved [10]. In our patient, although the initial MRI had confirmed a parietal stroke, this diagnosis alone did not explain the patient's presentation of whole-left sided facial droop more consistent with a peripheral lesion. Therefore, in addition to stroke, our differential diagnosis for the patient's facial paralysis upon admission included Bell's palsy, seizure with Todd's paralysis, and complex migraine.

Rare pontine strokes that affect the ipsilateral facial nerve nucleus or facial nerve tract before it exits the pons have been shown to represent a central lesion that presents identically to a peripheral lesion [11-13]. These insults occur acutely, whereas the onset of Bell's palsy typically progresses over multiple hours to days and would not be accompanied by nausea, vomiting, dizziness and diplopia [1]. Regarding the patient's etiology of his stroke, punctate infarcts that arise within the vascular territory of larger strokes have a greater likelihood of resulting from a proximal embolism. The patient was found to have infarcts in the parietal lobe and the pons, which are supplied by the middle cerebral artery and the vertebra-basilar system, respectively. Therefore, the patient's infarcts occurred in regions of the brain that utilize different vascular supplies, and his stroke was more likely related to small vessel disease than embolic in nature $[9,14]$. This aligns with the patient's increased risk for diffuse vascular disease given his past medical history of prior stroke, poorly controlled hypertension and hyperlipidemia.

The physical exam of patients with unilateral facial weak- ness should include evaluation of the external ear for vesicles or scabbing, which could indicate zoster. The parotid gland should be palpated for masses that could affect the nerve as it passes through the parotid gland. A history of facial twitching or spasms prior to the onset of weakness should prompt evaluation for parotid tumor lesions [1]. If the patient presents with bilateral facial palsy or clinical suspicion of Lyme disease, serologic testing following the two-tier testing of enzyme-linked immunosorbent assay (ELISA) and immunofluorescence assay (IFA) should be conducted. Any sign of nervous system dysfunction outside of cranial nerve seven e.g. dizziness, nystagmus or poor extraocular movement, and sensory loss should prompt evaluation for stroke or other neurovascular processes since Bell's palsy is an isolated facial nerve lesion by definition.

\section{Conclusions}

Searching for a subtle stroke on CT or MRI is comparable to combing for a needle in a haystack. Nevertheless, following intensive review of this patient's MRI images by practitioners who were familiar with his clinical presentation, an occult stroke was discovered. This emphasizes the importance of correlating a patient's radiographic imaging with their clinical presentation, which serves as a roadmap guiding the physician to the stroke's probable location. Physicians should not hastily abandon their clinical judgment when opposed by the initial radiologic evidence, yet should instead work with radiology to further investigate the image with the patient's clinical context in mind. Whilst the advent of imaging revolutionized medicine, we must be cautious to avoid total reliance upon it and continue to critically evaluate cases that contradict our suspicions. Ultimately, doctors must maintain high suspicion for a central lesion exhibiting peripheral neurological deficits before defaulting to a diagnosis of exclusion, treating accompanying warning symptoms e.g. refractory dizziness and labile blood pressures with high regard.

\section{Acknowledgments}

Not applicable.

\section{Informed Consent}

Not applicable.

\section{Financial Disclosure}

No funding was required.

\section{Conflict of Interest}

All the authors of this manuscript have no conflicts of interest 
to disclose.

\section{Author Contributions}

All authors had access to the data and a role in writing the manuscript, article type, key words, and running head.

\section{Abbreviations}

ADC: apparent diffusion coefficient; AICA: anterior inferior cerebellar artery; CT: computed tomography; DWI: diffusion weighted imaging; ECG: electrocardiogram; ED: emergency department; EMG: electromyography; ICU: intensive care unit; MRI: magnetic resonance imaging; PICA: posterior inferior cerebellar artery; TTE: transthoracic echocardiogram

\section{References}

1. Gilden DH. Clinical practice. Bell's Palsy. N Engl J Med. 2004;351(13):1323-1331.

2. Riga M, Kefalidis G, Danielides V. The role of diabetes mellitus in the clinical presentation and prognosis of Bell palsy. J Am Board Fam Med. 2012;25(6):819-826.

3. House JW, Brackmann DE. Facial nerve grading system. Otolaryngol Head Neck Surg. 1985;93(2):146-147.

4. Peitersen E. Bell's palsy: the spontaneous course of 2,500 peripheral facial nerve palsies of different etiologies. Acta
Otolaryngol Suppl. 2002;549:4-30.

5. Fahimi J, Navi BB, Kamel H. Potential misdiagnoses of Bell's palsy in the emergency department. Ann Emerg Med. 2014;63(4):428-434.

6. Kumral E, Bayulkem G, Evyapan D. Clinical spectrum of pontine infarction. Clinical-MRI correlations. J Neurol. 2002;249(12):1659-1670.

7. Pessin MS, Gorelick PB, Kwan ES, Caplan LR. Basilar artery stenosis: middle and distal segments. Neurology. 1987;37(11):1742-1746.

8. Xia C, Chen HS, Wu SW, Xu WH. Etiology of isolated pontine infarctions: a study based on high-resolution MRI and brain small vessel disease scores. BMC Neurol. 2017;17(1):216.

9. Mavrikakis I. Facial nerve palsy: anatomy, etiology, evaluation, and management. Orbit. 2008;27(6):466-474.

10. Blumenfeld H. Neuroanatomy through clinical cases, 2nd edition. Sinauer Associates: Sunderland, Mass. 2010.

11. Sherman SC, Thompson TM. Pontine hemorrhage presenting as an isolated facial nerve palsy. Ann Emerg Med. 2005;46(1):64-66.

12. Agarwal R, Manandhar L, Saluja P, Grandhi B. Pontine stroke presenting as isolated facial nerve palsy mimicking Bell's palsy: a case report. J Med Case Rep. 2011;5:287.

13. Bassetti C, Bogousslavsky J, Barth A, Regli F. Isolated infarcts of the pons. Neurology. 1996;46(1):165-175.

14. Oliveira-Filho J, Ay H, Shoamanesh A, et al. Abstract T MP58: Incidence and etiology of punctate infarcts in ischemic stroke patients. Stroke. 2018;46:ATMP58. 